never analysed the process in detail, Wright ${ }^{6}$ asserted that selection operates at the level of the species, and even Darwin's Origin of Species alludes to this process.

Department of Earth and

Steven M. Stanley

Planetary Sciences,

The Johns Hopkins University,

Baltimore, Maryland 21218, USA

1. Maynard Smith, J. Nature 330, 516 (1987)

2. Maynard Smith, J. Nature 332, 311-312 (1988).

3. Stanley. S.M. Macroevolution (Freeman. San Francisco, 1979)

4. Stanley. S.M. Evolution 36, 460-473 (1982)

5. Stanley. S.M. Proc. natn. Acad. Sci. U.S.A. 72, 646 (1975) 6. Wright, S. Am. Nat. 90, 5-24 (1956)

\section{Unusual segregation of cystic fibrosis allele to males}

SIR-One in fifty chromosomes seven in caucasian populations has the cystic fibrosis (CF) mutant gene. A strong linkage disequilbrium has recently been described with the DNA probes KM-19, CS-7 and $\mathrm{XV}-2 \mathrm{c}^{1-3}$, suggesting that most CF chromosomes in most European populations are derived from a single mutation event. It is not clear whether the mutation expanded through some form of carrier advantage, or by founder effect followed by genetic drift. It has been suggested that the high frequency of the CF gene could have arisen through increased fertility of $\mathrm{CF}$ carriers ${ }^{4.5}$; recently Pritchard ${ }^{6}$ reported a slight but unexpected increase of children in the families of uncles of CF children. $\mathrm{He}$ also reported an increase in the proportion of male children in these families.

To investigate the significance of that observation in a definitive way we have undertaken the haplotyping of asymptomatic CF carriers by DNA analysis. We investigated 50 French families, each with a CF individual, in which there was also at least one normal sibling, to determine whether the sibling is homozygous for the unaffected allele, or a CF carrier. Among the 60 siblings investigated, who include about the same number of boys and girls, we observe a striking distortion of segregation with sex. Twenty of 22 normal homozygotes $(91 \%)$ are girls $\left(\chi^{2}=18.1\right.$, $P<0.0001)$, and 16 of $21(76 \%)$ heterozygotes carrying the paternal CF chromosome are boys $\left(\chi^{2}=5.76, P<0.02\right)$. In contrast, the maternal $\mathrm{CF}$ chromosome has been passed equally to boys and girls.

\section{Scientific Correspondence}

Scientific Correspondence is intended to provide a forum in which readers may raise points of a scientific character. They need not arise out of anything published in Nature. In any case, priority will be given to letters of less than 500 words and five references.
This unexpected result arises from the fact that $\mathrm{CF}$ chromosomes of paternal origin, unlike the normal chromosomes, are passed preferentially to males. Note that, as previously reported, the overall proportion of carriers to homozygous normals is $\sim 2$ to 1 , as expected ${ }^{3}$.

We hope that others will test families in this way to determine whether this result is statistical chance, or can be confirmed in other populations. If generally true, it suggests a mechanism by which the $\mathrm{CF}$ gene pool can be expanded through preferential inheritance of the affected chromosomes from male to male. Such a mechanism could involve close linkage of $\mathrm{CF}$ to some determinant of sperm viability or fitness, or selection during early embryonic development. It also suggests that the proportion of $\mathrm{CF}$ carriers will be higher in males than in females.

A. Kitzis

J. C. ChOMEL

J. C. KAPLAN

Institut de Pathologie Moleculaire, CHU Cochin, 75014 Paris, France

G. Giraud

A. LABBE

CHU Clermont-Ferrand, France

B. Dastugue

V. DuMur

J. P. Farriaux

Ph. Roussel

CHU Lille, France

R. Williamson

St Mary's Hospital Medical School,

London W2 IPG, UK

INSERM U 155, Paris, France

J. FEINGOLD

1. Estivill, X. et al. Nature 326, 840-845 (1987)

2. Estivill, X. et al. Genomics 1, 257-263 (1987).

. Beaudet, A. L. New Engl. J. Med. 318, 50-51 (1988).

Danks, D. M. Allan, J. \& Anderson, C. M. Ann. hum Genet, 28, 323-335 (1965)

Knudson, A. G. Jr, Wayne, L. \& Hallett, C. M. Am. J. hum. Genet. 19, 388-392 (1967).

6. Pritchard, D. J. Nature 330, 319 (1987)

\section{'Cytoplasmic' and 'maternal' inheritance}

SIR-Altenberg's attempt (Nature 331, $309 ; 1988)$ to clear up the confusion between the use of 'maternal inheritance' and 'maternal effect' is helpful but still leaves unnecessary confusion. There is agreement that when the genotype of the maternal parent determines the phenotype of the progeny regardless of the progeny's genotype (as in the classic example of shell coiling in Lymnaea peregra snails discussed by John Galloway Nature 330, 204; 1987), the appropriate term is 'maternal effect'.

Confusion is introduced by using the term 'maternal inheritance' for a phenomenon which should more appropriately be called 'cytoplasmic inheritance'. In higher organisms, where the egg cell is the only source of extranuclear hereditary factors, the term 'maternal inheritance' is accurate. The term 'cytoplasmic inheri- tance' is more appropriate because it also describes the identical phenomenon in organisms such as protozoa where use of 'maternal' would not be correct, and it avoids the linguistic confusion of 'maternal effect' and 'maternal inheritance'

This is subtly recognized in the dictionary definition of maternal inheritance which indicates that the phenomenon is also called cytoplasmic inheritance, although there is no analogous qualification under the definition of 'cytoplasmic inheritance' (R.C. King \& W.D. Stansfield, A Dictionary of Genetics; Oxford University Press, 1985). The use of the term 'maternal inheritance' is an unnecessary and confusing representation of the underlying biological basis of the inheritance pattern being described.

Bruce Haggard

Hendrix College,

Conway, Arkansas 72032, USA

\section{HIV and HLA similarity}

SIR-Recent data suggest a functional role for a conserved region of the human immunodeficiency virus (HIV) envelope glycoprotein gp120: mutations introduced into this sequence ${ }^{1}$ and antibodies directed against the concerned region ${ }^{2}$ block virus infectivity without affecting virus binding to the CD4 receptor. I would like to point out that this region has a striking resemblance to a 10 -amino-acid region in the extracellular, membrane-proximal domain of HLA class II $\beta$-chains (see figure).

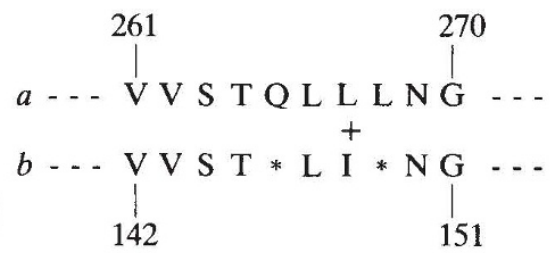

Comparison of HIV gp120 $(a)$ and the consensus HLA-DR, -DP and -DQ $\beta$-chain $(b)$ sequences. Stars, dissimilar residues. Plus sign, a conservative change.

HLA class II molecules and gp120 are both recognized by membrane components of CD4-positive T lymphocytes. In both cases ${ }^{1.3}$, the 10 -amino-acid region is distant in the primary amino-acid sequence from the predicted CD4-binding sites. It is conceivable that this short sequence contributes to both antigen presentation and virus infection by interacting with an as yet undefined component of the T-cell membrane, distinct from the CD4 molecule. If so, HIV could have evolved to exploit more than one T-cell component of the antigen presentation machinery to infect its target cells.

JoHn A.T. YounG University of California Medical School, San Francisco, California 94143, USA

1. Willey, R.L. et al. J. Virol. 62, $139-147$ (1988).
2. Ho, D.D. et al. Science 239, 1021-1023(1988).
3. Golding, H. et al. Nature 317, $425-427$ (1985). 